\title{
Das RAAS ist ein Schlüssel zur Risikoreduktion
}

\author{
Ein Schlüssel zur Organprotektion \\ liegt im Eingriff in das Renin-Angio- \\ tensin-System. Immer mehr Studien \\ bestätigen das, für ACE-Hemmer, \\ für Angiotensin-II-Rezeptor-Anta- \\ gonisten und auch für Aliskiren.
}

Das Renin-Angiotensin-System ist zu einem zentralen Thema der Herz-Kreislauf-Forschung geworden. Angiotensin II im Übermaß wird eine Schlüsselbedeutung beim Fortschreiten der kardiovaskulären Erkrankungen zugeschrieben - von der Hypertonie bis zum Organversagen. Mit Hemmstoffen von Angiotensin II werden immer neue Studien veröffentlicht, die den Schutz von Hirn, Herz und Nieren belegen. An Eingriffe ins Renin-Angiotensin-System knüpfen sich große Erwartungen für den Organschutz. Belegt wurde dieser zunächst mit einigen ACE-Hemmern.

\section{Sartane haben praktisch keine unerwünschten Wirkungen}

Dass ein Sartan mindestens so wirksam ist wie ACE-Hemmer, wurde zunächst in der Groß-Studie ONTARGET mit Telmisartan im Vergleich zu Ramipril bestätigt. Der Vorteil der Sartane ist, dass sie praktisch keine unerwünschten Wirkungen haben.

\section{Daten belegen Schutz}

Zum Organschutz von AngiotensinII-Antagonisten gibt es viele Daten für Patienten mit Herzinsuffizienz, mit dia betischer Nephropathie, nach Herzinfarkt und zum Schutz vor Insulten. Dass die Progression der Nephropathie mit Sartanen gehemmt wird, ist vielfach belegt, zum Beispiel für Irbesartan, Losartan, Telmisartan und Valsartan. Für Olmesartan bestätigen Daten, dass eine Mikroalbuminurie gebremst wird. Auch der Renin-Hemmer Aliskiren hat in einer Studie renoprotektive Wirkeigenschaften bewiesen.

Zum Herzschutz trägt die Hemmung einer linksventrikulären Hypertrophie durch Sartane bei. So reduziert Losartan, das es inzwischen generisch gibt, effizient das Auftreten von Vorhof flimmern im Vergleich zu einem Betablocker. Und Valsartan wirkt protektiv nach Herzinfarkt. Neuroprotektion, die über die Blutdrucksenkung hinausgeht, ist etwa belegt mit Eprosartan sowie mit Losartan und mit Candesartan.

(Rö) $\rightarrow$ senkung unter $120 \mathrm{mmHg}$ versus unter $140 \mathrm{mmHg}$ verglichen.

\section{„Hochnormal" ist normal}

Auch die nun empfohlenen nicht mehr ganz so niedrigen Zielwerte lassen sich in den allermeisten Fällen nur mit einer blutdrucksenkenden Kombinationstherapie erreichen. Des weiteren wird der Beginn einer antihypertensiven medikamentösen Therapie bereits bei hochnormalem Blutdruck, wie noch in den Leitlinien aus dem Jahr 2007 empfohlen, nicht mehr favorisiert. Denn es fehlt die Evidenz aus prospektiven Studien.

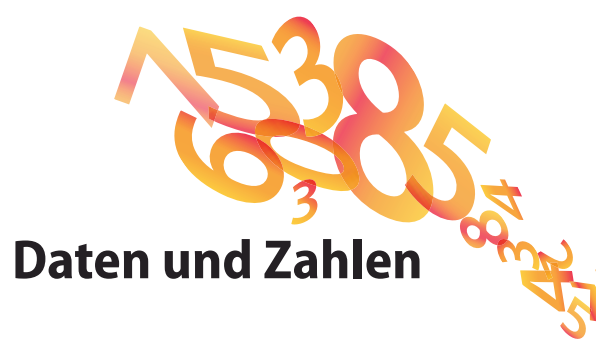

Nur jeder dritte behandelte Hypertoniker erreicht seinen Zielwert. Das ist trotz der guten Therapiemöglichkeiten immer noch vielfach die Realität.

Eine fixe Dreierkombination ist mit Valsartan/Amlodipin/HCT in verschiedenen Dosierungen im vergangenen Jahr auf den Markt gekommen. Bereits in den 70er Jahren waren solche Kombinationen - allerdings mit anderen Substanzen - üblich.

$5 \mathbf{~ m m H g}$-Änderungen des diastolischen Blutdruckes gehen mit einer etwa 20\%igen Veränderung des relativen Risikos für koronare Ereignisse einher. Das relative Risiko, einen Schlaganfall zu erleiden, verändert sich um 35\%.

Die Hypertonie gewinnt auch als Risikofaktor für Demenzen zunehmend an Bedeutung. Das gilt sowohl für Demenzen vom vaskulären Typ als auch für Demenzen vom Alzheimer-Typ.

\section{Infos im Internet}

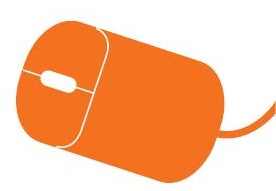

Alle Folgen der Sommer-

Akademie 2010 finden Sie im Web unter www.aerztezeitung.de

Informationen, Adressen und Leitlinien - die Deutsche Hochdruckliga ist das Kompetenzzentrum. www.hochdruckliga.de

Die Europäische Hochdruckgesellschaft hat die Leitlinien aktualisiert.

www.eshonline.org 